\title{
Tensor Product based modeling of Tumor Growth
}

\author{
György Eigner*, Imre Rudas ${ }^{\dagger}$, Anikó Szakál ${ }^{\dagger}$, Levente Kovács * \\ *Physiological Controls Research Center and Research, Innovation, and Service Center, \\ Óbuda University, Budapest, Hungary, \\ Email: \{eigner.gyorgy,kovacs.levente\}@nik.uni-obuda.hu \\ ${ }^{\dagger}$ Research, Innovation, and Service Center, \\ Óbuda University, Budapest, Hungary \\ Email: \{rudas,szakal\}@uni-obuda.hu
}

\begin{abstract}
The application of the Soft Computing based methods, especially, the Tensor Product (TP) transformation has several beneficial properties from the biological modeling and control point of view, because complex, nonlinear processes can be handled by them effectively. Another advantage of these tools consist on the Linear Parameter Varying (LPV) and Linear Matrix Inequality (LMI) based techniques can be easily connected to them. The aim of this study is to develop TP models, which can describe the tumor growth beside anti-angiogenic treatment. The role of the anti-angiogenic therapies is to decrease the size of the tumor to operable or maintainable level. From control engineering point of view, the treatment process can be formulated as a control task. In this work, we realized two TP models, which approximates the initial transformed model with high accuracy, regardless the kind of input load and without stability problems. The TP models will be used for TP-based controller design on LMI basis.
\end{abstract}

Index Terms-Anti-angiogenic therapy, Tumor growth model, Tensor Product model transformation, TP-based modeling

\section{INTRODUCTION}

Beside the well known classical cancer therapies as radiotherapy, chemotherapy and surgical intervention, one of the modern treatment directions are the Targeted Molecular Therapy (TMT). In case of TMT, different drugs and/or other substances are used to block or eliminate the growth and spread of the cancer by interfering with specific molecules which play important roles in biological processes regard to the growth, progression and spread of the cancer. To sum up, the aim is the inhibition of certain processes and not to kill the tumor itself. Compared to the classical treatments, the main benefits of them are the limited side effects and the more focused therapy. Several TMTs exist, although, the most important ones are the apoptosis inducers, signal transmission inhibitors, gene expression modulators and anti-angiogenic therapies [1].

In case of the anti-angiogenic therapies the goal of the TMT is to inhibit the growing of the supplying vasculature of the cancer. It is known, that the supplying vasculature is needed to provide the oxygen and nutrients after the tumor

Gy. Eigner was supported by the ÚNKP-16-3/IV. New National Excellence Program of the Ministry of Human Capacities. This project has received funding from the European Research Council (ERC) under the European Union's Horizon 2020 research and innovation programme (grant agreement No 679681) grows beyond a certain volume. The limit coming from the local diffusion, which does not satisfy the nutrition needed for further growth or to maintain the reached size. By blocking the formation of new blood vessels - which can cover the nutrition of the tumor - the size of the affected cell population can be decreased or kept below a given level which is bearable from the human body point of view. Normally, the angiogenec processes are infrequent in healthy adults, but frequent regard to tumor growth and by applying inhibition only limited side effects occur compared to the regular therapies.

The angiogenesis is regulated by pro- and anti-angiogen factors. The most important pro-angiogen factor is the vascular endothelial growth factor (VEGF). The VEGF regulates the endothelial proliferation, namely, reproduction of endothelial cells which form the blood vessels - that makes it excellent target for such inhibitor therapy [1]-[3].

The anti-angiogenic therapies are mostly used beside regular therapies, such as chemotherapy and/or radiotherapy. Although, the application of them as monotherapy was considered recently, there are several open questions regarding to the appropriate drug dosage protocols [4]. In clinical practice, three approach are used for drug delivery: bolus doses therapy (BDT), metronomic low dose therapy (MLDT) and continuous infusion therapy (CIT). In the first case, the amount of the injected drug is mostly the maximum tolerable dosage and between the boluses there are no anti-angiogenic kind drug intake. The main drawbacks are the higher occurrence of side effects, moreover, the remaining tumor cells may become resistant to the therapy because of their fast evolution and proliferation [5]. In order to avoid these unfavorable effects, the MLDT can be used, in which case the anti-tumor drugs are delivered in minimal dosage based on strict schedule over longer periods [6]. Investigations concerning to the application of CIT (based on animal- and in-silico-experiments) recently showed that this can be the most effective treatment among the current anti-cancer therapies [7]-[9]. Although, in order to apply such kind of protocol, highly advanced biomedical modeling and controller design tools are needed which can efficiently handle the challenges regard this field, for example intra- and inter-patient variability, nonlinearities and so on.

In the last twenty years, several possible theorems and tools were developed to handle the aforementioned issues. One 
of these is the Linear Paramter Varying (LPV) methodology which allows to use linear controller design theorems in case of nonlinear systems by enclosing the nonlinearities into internal bounded variables [10], [11]. The Robust Fixed Point Theorem (RFPT) based controller design also suitable for biological related controls, because of its flexibility and inverse approach [12], [13]. Advanced Soft Computing (SC) based methods can be appropriate solutions as well [14]. As the part of the SC, the recently developed TP-based modeling and control provides various beneficial tools, eg. transforms the nonlinear models into linear ones with high accuracy. The main goal of TP model transformation is to develop TPmodel objects, which are ready for TP-based controller design. The resulting TP model and controller structures includes the uncertainties as a hidden way - moreover, LMI based techniques can be combined with them [15], [16].

In this study, we developed two TP model structures which approximate the initial transformed model with high accuracy and can be used for TP-based controller design.

The paper is structured in the following way: firstly the applied original and transformed tumor growth model were introduced. After, the TP model transformation was presented. In Sec. IV., we introduced the qLPV models and the developed TP models which is followed by the validation of them. Finally, our findings were presented.

\section{InVestigated Tumor Growth Model}

The examined tumor growth model under angiogenic inhibition originates from the well-known Hahnfeldt model [17]. This model describes the growing dynamics of the tumor and supporting vasculature over $t$ time as follows:

$$
\begin{gathered}
\dot{x}_{1}(t)=-\lambda_{1} x_{1}(t) \log \left(\frac{x_{1}(t)}{x_{2}(t)}\right), \\
\dot{x}_{2}(t)=b x_{1}(t)-d x_{1}^{2 / 3}(t) x_{2}(t)-\eta x_{2}(t) g(t), \\
\dot{g}(t)=-\lambda_{3} g(t)+u(t),
\end{gathered}
$$

where $x_{1}(t)[\mathrm{mm}]^{3}$ and $x_{2}(t)[\mathrm{mm}]^{3}$ are the volume of the tumor and supporting vasculature, respectively and $g(t)$ $[\mathrm{mg} / \mathrm{kg}]$ describes the inhibitor serum level in time. The output of the model is the measurable state, $x_{1}(t)$. The belonging model parameters are $\lambda_{1}=0.19211 /$ day, $b=5.851$ $1 /$ day, $d=0.008711 /\left(\mathrm{mm}^{2}\right.$ day $), \eta=0.66 \mathrm{~kg} /(\mathrm{mg}$ day $)$ and $\lambda_{3}=1.31$ 1/day - these model parameters coming from [17] based on mice experiments (tumor: Lewis lung carcinoma, inhibitor: endostatin). The model contains multiple nonlinearities which have to be handled in practice. Although, according to [17] there is strict limitation concerning to the states, namely $x_{1}(t), x_{2}(t)>\forall t(t>0)$, feasibility problems can be occurred due to the $\log \left(\frac{x_{1}(t)}{x_{2}(t)}\right)$ term in (1) - (0/0) type singularity can be occurred, if $x_{1}(t)$ and $x_{2}(t)$ are equal to zero and numerical stability problems may appear, if $x_{1}(t)$ and $x_{2}(t)$ are close to zero. In order to handle these kind of limitations, mathematical transformations can be applied such as [18], [19]. In this work we used the transformed version of the original model presented by [19]. By introducing the $y_{1}(t)=\log \left(x_{1}(t)\right)[\mathrm{mm}]^{3}$ and $y_{2}(t)=\log \left(x_{2}(t)\right)[\mathrm{mm}]^{3}$ new state variables, the following transformed model occurs:

$$
\begin{gathered}
\dot{y}_{1}(t)=-\lambda_{1} y_{1}(t)+\lambda_{1} y_{2}(t), \\
\dot{y}_{2}(t)=b e^{y_{1}(t)-y_{2}(t)}-d e^{2 y_{1}(t) / 3}-\eta g(t), \\
\dot{g}(t)=-\lambda_{3} g(t)+u(t) .
\end{gathered}
$$

The nontrivial equilibrium of the original model ((1) and (2)) was described in [8], in which was proven that beside constant inhibitor level $g(t) \equiv g_{\infty}$ the $x_{1, \infty}$ and $x_{2, \infty}$ can be calculated as follows:

$$
x_{1, \infty}=x_{2, \infty}=\left(\frac{b-\eta g_{\infty}}{d}\right)^{3 / 2} .
$$

From (7) it is clear that $x_{1, \max }=x_{2, \max }=\left(\frac{b}{d}\right)^{3 / 2}$, if the inhibitor level is equal to zero $(g=0)$. Thus, considering the limitations and (7), the operating domain becomes: $x_{1}(t)=$ $x_{2}(t)=\left(0,(b / d)^{3 / 2}\right]$.

The aim of the anti-angiogenic therapy is to reach lower tumor volume via inhibition of its angiogenesis in order to make it operable or maintainable - however, the volume of the tumor cannot be totally eliminated only by anti-angiogenic therapy [3]. From this consideration, it is reasonable to select a higher lower limit for $x_{1}$ and $x_{2}$, which also leads to a more manageable transformed model from mathematical point of view as well. Assume the following domain for $x_{1}(t)=x_{2}(t)=\left[1,(b / d)^{3 / 2}\right]$, which consequences that the domain of the transformed states become $y_{1}(t)=y_{2}(t)=$ $\left[\log (1), \log \left((b / d)^{3 / 2}\right)\right]$. In order to make the TP models numerically stable, we applied an other restriction regard to the lower limit of the investigated domain, which will be detailed in Sec. IV. In this work we consider this new domain for the $y_{1}(t)$ and $y_{2}(t)$ transformed states.

The deep investigation of such limitation and the transformed model can be found in [19].

\section{Tensor Product Model Transformation}

The TP modeling originates from the Fuzzy System (FS) theorems. More precisely, the Takagi-Sugeno FS (TSFS) is able to describe a system model given by its quasi-LPV (qLPV) state space representation, if the universe of the FS (whereon the membership functions are defined) is the parameter vector of the qLPV model [20]. The TP model transformation has several beneficial properties compared to the TSFS - the most beneficial is that the TP models describe the original models with similar precision as the TSFS, but the parameter domain of them can be much more tighter than the TSFS's domain.

The TP model transformation transforms a given qLPV function into a TP model structure. Because of the qLPV models (as state space representation) can be represented by qLPV functions, the TP transformation can be easily executed [16]. The occurring TP model is a multidimensional tensor product structure consists of convex combination of a 
high-order core tensor and different weighting functions in appropriate dimensions belong to the parameter vector [15]. The resulting TP models - thankfully the convex hull manipulation - realizes convex polytopic structures, which allows to combine the transformation with LMI-based techniques [16]. The approximation accuracy of the TP model is determined by the number of samples in the parameter domain, namely, the vertices of the polytopic structure.

A general qLPV model can be written as follows:

$$
\begin{aligned}
& \dot{\mathbf{x}}(t)=\mathbf{A}(\mathbf{p}(t)) \mathbf{x}(t)+\mathbf{B}(\mathbf{p}(t)) \mathbf{u}(t) \\
& \mathbf{y}(t)=\mathbf{C}(\mathbf{p}(t)) \mathbf{x}(t)+\mathbf{D}(\mathbf{p}(t)) \mathbf{u}(t)
\end{aligned}
$$

where the matrices $\mathbf{A}(\mathbf{p}(t)) \in \mathbb{R}^{k \times k}, \mathbf{B}(\mathbf{p}(t)) \in \mathbb{R}^{k \times m}$, $\mathbf{C}(\mathbf{p}(t)) \in \mathbb{R}^{l \times k}$, and $\mathbf{D}(\mathbf{p}(t)) \in \mathbb{R}^{l \times m}$ represent the state-, input-, output- and forward-matrices, respectively. The $\mathbf{x}(t) \in$ $\mathbb{R}^{k}$ is the state vector, $\mathbf{u}(t) \in \mathbb{R}^{m}$ is the input vector and $\mathbf{y}(t) \in \mathbb{R}^{l}$ is the output vector. The $\mathbf{p}(t) \in \Omega \in \mathbb{R}^{N}$ is the time dependent parameter vector - which consists of $N$ scheduling variables $p_{i}(t) i=[1, . ., N]$.

$$
\mathbf{S}(\mathbf{p}(t))=\left(\begin{array}{ll}
\mathbf{A}(\mathbf{p}(t)) & \mathbf{B}(\mathbf{p}(t)) \\
\mathbf{C}(\mathbf{p}(t)) & \mathbf{D}(\mathbf{p}(t))
\end{array}\right)
$$

where the parameter dependent complex $\mathbf{S}(\mathbf{p}(t)) \in$ $\mathbb{R}^{(k+l) \times(k+m)}$ represents the qLPV function (system matrix).

The parameter vector $\mathbf{p}(t) \in \Omega \in \mathbb{R}^{N}$ is enclosed into the $\Omega$ domain, where $\Omega=\left[p_{1, \text { min }}, p_{1, \max }\right] \times\left[p_{2, \min }, p_{2, \max }\right] \times$ $\ldots \times\left[p_{N, \min }, p_{N, \max }\right] \in \mathbb{R}^{N}$ formalizes a limited hypercube in the $N$-dimensional hyperspace - which is determined by the extremes of the scheduling variables [15], [16].

The finite element polytopic TP model approximates $\mathbf{S}(\mathbf{p}(t))$ inside the closed hypercube in the following way:

$$
\mathbf{S}(\mathbf{p}(t)) \equiv \sum_{r=1}^{R} w_{r}(p(t)) \mathbf{S}_{r}
$$

where $w_{r}(p(t))$ are the parameter dependent weighting functions and $\mathbf{S}_{r}$ is the core tensor.

In other way, the $\mathbf{S}(\mathbf{p}(t))$ can be described as a linear combination of convex weighting functions and the LTI vertex system for each $\mathbf{p}(t) \in \Omega$, which results convex combination [16], [21]. Accordingly, - through applying a sampling on the parameter space - the TP based polytopic finite element model can be described as follows [16], [22]:

$$
\mathbf{S}(\mathbf{p}(t))=\sum_{i_{1}=1}^{I_{1}} \sum_{i_{2}=1}^{I_{2}} \ldots \sum_{i_{N}=1}^{I_{N}} \prod_{n=1}^{N} w_{n, i_{n}}\left(p_{n}(t)\right) \mathbf{S}_{i_{1}, i_{2}, \ldots, i_{N}} .
$$

The (11) can be written in the following TP form:

$$
\mathbf{S}(\mathbf{p}(t))=S \underset{\mathrm{n}=1}{\mathrm{~N}} \mathbf{w}_{n}\left(p_{n}(t)\right)
$$

where $S$ core tensor - built up from the $\mathbf{S}_{i_{1}, i_{2}, \ldots, i_{N}}$ LTI vertex system - contains the system coefficients in all dimensions as $S \in \mathbb{R}^{I_{1} \times I_{2} \times \ldots \times I_{N} \times(k+l) \times(k+m)}$, further, the $\mathbf{w}_{n}\left(p_{n}(t)\right)$ vector consist of the continuous convex weighting functions $w_{n, i_{n}}\left(p_{n}(t)\right)\left(i_{n}=1 \ldots I_{N}\right)$. The aforementioned convexity criteria is satisfied regarding to the weighting functions and via the TP model as well, if the following statements are true:

$$
\begin{aligned}
& \forall n, i, p_{n}(t): w_{n, i_{n}}\left(p_{n}(t)\right) \in[0,1] \\
& \forall n, p_{n}(t): \sum_{i=1}^{I_{n}} w_{n, i_{n}}\left(p_{n}(t)\right)=1
\end{aligned} .
$$

Different convex hulls can be applied during the TP model transformation depends on the goals, eg. extended or tight operating domain [16]. In this work, we applied the a Minimal Volume Simplex (MVS) kind convex hull [15], [21] for the TP type polytopic qLPV model, which is able to provide a tight operating domain in the parameter space. The TP model wellapproximates the original model inside the $\Omega$ hypercube and the volume of the $\Omega$ is as low as possible. In this way, the TP model with MVS type convex hull can be described as:

$$
\mathbf{S}(\mathbf{p})=S \underset{\mathrm{n}=1}{\mathrm{~N}} \mathbf{w}^{(n)}\left(p_{n}\right),
$$

where $S \in \mathbb{S}^{J_{1} \times \ldots \times J_{N}}$ core tensor is created from the $\mathbf{S}_{j_{1}, \ldots, j_{N}}$ vertices. In this way, the $(S)_{j_{n}=j} n$-mode sub-tensors realizes a minimal volume bounding hypercube for the $S \times{ }_{n} \mathbf{w}_{j n}^{(n)}\left(p_{n}\right)$ trajectory over $n=1 . . N$. Further details and explanations can be found in [15], [16], [21], [23].

\section{QLPV- AND TP-MODELS}

During the examinations, we used the mentioned transformed model from (4), (5) and (6). Since, our future goal is to use the developed models for control purposes, we investigated two possible qLPV model realizations and via TP model descriptions. Due to the fact, that the transformed model contains nonlinearities only in (5), these have to be selected as scheduling variables.

The selected terms from (5) were $p_{1}(t)=e^{y_{1}(t)-y_{2}(t)}$ and $p_{2}(t)=e^{2 y_{1} / 3}$, which means $\mathbf{p}(t)=\left[p_{1}(t), p_{2}(t)\right]^{\top}$. These selections modify the (5) as follows:

$$
\dot{y}_{2}(t)=b p_{1}(t)-d p_{2}(t)-\eta g(t) .
$$

To realize a qLPV model, both scheduling variables have to be paired to a given state in order to involve them into the qLPV function - although, this can be done in multiple ways. In this study, we investigated two cases which requires the extension of $p_{1}$ and $p_{2}$ as follows:

- Model I (qLPV): $p_{1}(t)=\frac{e^{y_{1}(t)-y_{2}(t)}}{y_{1}(t)}$ and $p_{2}(t)=\frac{e^{2 y_{1} / 3}}{y_{2}(t)}$

- Model II (qLPV): $p_{1}(t)=\frac{e^{y_{1}(t)-y_{2}(t)}}{y_{2}(t)}$ and $p_{2}(t)=$ $\frac{e^{2 y_{1} / 3}}{y_{1}(t)}$

In both cases, the redefinition of the operating domain of the states are needed in order to avoid the singularity. Due to this fact, instead of the $y_{1}(t)=y_{2}(t)=\left[\log (1), \log \left((b / d)^{3 / 2}\right)\right]$ domain at least the following tighter lower limit should be considered: $y_{1}(t)=y_{2}(t)=\left(\log (1), \log \left((b / d)^{3 / 2}\right)\right]-$ which is in accordance that the phenomena and the possible control goals [8]. It has to be mentioned, that the lower limit can 
be as low as possible to avoid the singularity, however, does not cause numerical problems. Although, we applied $\log (2)$, thus $y_{1}(t)=y_{2}(t)=\left[\log (2), \log \left((b / d)^{3 / 2}\right)\right]$, which means $x_{1, \min }=x_{2, \min }=\log (2)=0.6931[\mathrm{~mm}]^{3}$. As a consequence, the developed TP models accurately describe the transformed model between these limits, moreover, the parameter domain became much more smaller which does not decrease the generality, but increase the numerical stability. For example, if the lower limit is $\log \left(1+10^{-5}\right)=10^{-5}$ the singularities can be avoided, but it results a much bigger parameter domain than if the lower limit is $\log (2)=0.6931$.

In case of Model I, the (15) has to be modified as follows, moreover, the qLPV function becomes:

$$
\dot{y}_{2}(t)=b p_{1}(t) y_{1}(t)-d p_{2}(t) y_{2}(t)-\eta g(t) .
$$

and

$$
\mathbf{S}(\mathbf{p}(t))=\left[\begin{array}{cccc}
-\lambda_{1} & \lambda_{1} & 0 & 0 \\
b p_{1}(t) & -d p_{2}(t) & -\eta & 0 \\
0 & 0 & -\lambda_{3} & 1 \\
1 & 0 & 0 & 0
\end{array}\right]
$$

Based on the mentioned domains of the states, the operating domain of the scheduling variables were $p_{1}(t)=[1.6548$. $\left.10^{-4}, 892.6771\right]$ and $p_{2}(t)=[0.1625,970.0880]$. We applied medium sampling: 555 in both domains, which was satisfying from the accuracy point of view. The TP model transformation was applied on (17), which results the following general TP model structure:

$$
\begin{aligned}
& \mathbf{S}\left(p_{1}(t), p_{2}(t)\right)=S \underset{\mathrm{n}=1}{\otimes} \mathbf{w}_{n}\left(p_{n}(t)\right)= \\
& S \times_{1} \mathbf{w}_{1}\left(p_{1}(t)\right) \times_{2} \mathbf{w}_{2}\left(p_{2}(t)\right)
\end{aligned}
$$

The vary of the applied MVS type weighting functions - which are linear in this case - can be seen on Fig. 1.

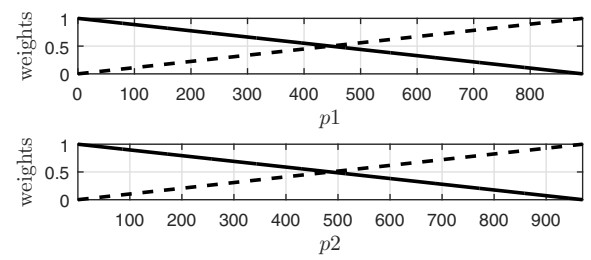

Figure 1. Weighting function belong to TP model version 2

In case of Model II, the (15) has to be modified in the following way:

$$
\dot{y}_{2}(t)=-d p_{2}(t) y_{1}(t)+b p_{1}(t) y_{2}(t)-\eta g(t) .
$$

and

$$
\mathbf{S}(\mathbf{p}(t))=\left[\begin{array}{cccc}
-\lambda_{1} & \lambda_{1} & 0 & 0 \\
-d p_{2}(t) & b p_{1}(t) & -\eta & 0 \\
0 & 0 & -\lambda_{3} & 1 \\
1 & 0 & 0 & 0
\end{array}\right] .
$$

Due to the symmetry, the operating domains of $p_{1}(t)=$ $\left[1.6548 \cdot 10^{-4}, 892.6771\right]$ and $p_{2}(t)=[0.1625,970.0880]$ were the same and the applied sampling was 555 in both domains.
The TP model transformation was applied on (20), which results the following general TP model structure as follows:

$$
\begin{aligned}
& \mathbf{S}\left(p_{1}(t), p_{2}(t)\right)=S \underset{\mathrm{n}=1}{\otimes} \mathbf{w}_{n}\left(p_{n}(t)\right)= \\
& S \times_{1} \mathbf{w}_{1}\left(p_{1}(t)\right) \times{ }_{2} \mathbf{w}_{2}\left(p_{2}(t)\right)
\end{aligned}
$$

The vary of the applied MVS type weighting functions - which are linear in this case as well - can be seen on Fig. 2.

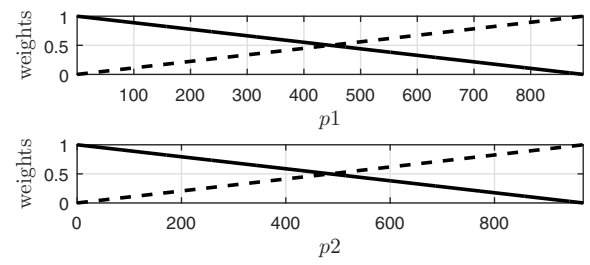

Figure 2. Weighting function belong to TP model version 2

\section{VALIDATION}

During the validation we compared the behavior of the transformed model to the developed TP models. We applied three scenarios which covered the most important intake protocols from applicable control strategy point of view. These were the followings:

- Natural answer of the models (without external input);

- Answer of the models beside continuous sinusoidal control input;

- Answer of the models beside impulse kind control input. In all cases we applied the following initial conditions: $y_{1}(0)=\log (10000), y_{2}(0)=\log (9950), g(0)=0$. In both cases, we simulated 100 virtual days.

The basis of the comparison was the $\mathcal{L}_{1}$ norm of the difference of the states of the transformed model and the developed TP models. Beside, the vary of the $\mathbf{p}(t)$ and the applied input signals (if any) were represented on the diagrams.

\section{A. Natural Answer of the Models}

The first investigated properties were the behavior of the TP models compared to the transformed model without external input. This property is important due to the internal instability of the original model originates from the phenomena. However, the transformed model and via the TP models have more convenient boundaries. During the simulations, both the transformed model and the TP models reach their steady states $\left(y_{i, \infty}=\log (b / d)^{3 / 2}=9.7663, i=1,2\right)$ started from their initial conditions $\left(y_{1}(0)=\log (10000), y_{2}(0)=\log (9950)\right)$ without stability problems. Due to external input was not applied, the $g(t)=0, \forall t \geq 0$.

As it can be seen (Fig. 3 and 4), the deviation dynamics were similar in case of the TP model I. and the TP model II. as well. Since the scheduling variables were symmetrical with the opposite dynamics.

It is visible on Fig. 3 and 4, the differences were only numerical $\left(\approx 10^{-10}\right)$ in both cases between the states of the transformed model and the TP models. 

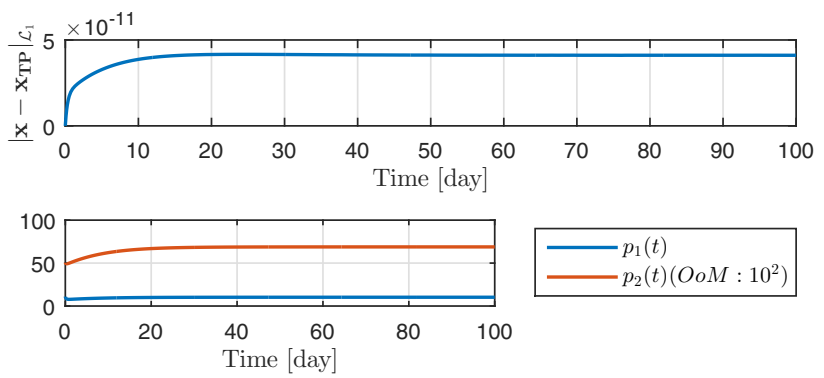

Figure 3. Upper Diagram: Comparison of the states of the transformed model and TP Model I. based on $\mathcal{L}_{1}$ norm, without external load (States: $\mathbf{x}(t)=$ $\left.\left[y_{1}(t), y_{2}(t), g(t)\right]^{\top}\right)$. Lower Diagram: Vary of scheduling parameters over time. OoM: Order of Magnitude.
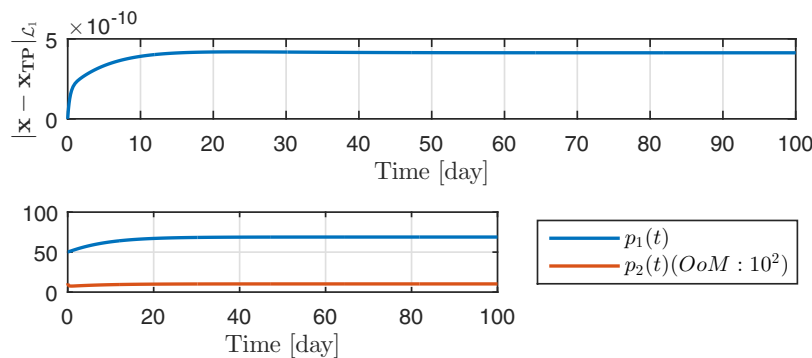

Figure 4. Upper Diagram: Comparison of the states of the transformed model and TP Model II. based on $\mathcal{L}_{1}$ norm, without external load (States: $\mathbf{x}(t)=$ $\left.\left[y_{1}(t), y_{2}(t), g(t)\right]^{\top}\right)$. Lower Diagram: Vary of scheduling parameters over time. OoM: Order of Magnitude.

\section{B. Answer of the Models beside Continuous Sinusoidal Con- trol Input}

In this scenario, we tested the long term stability of the models beside continuous, softly oscillating input. Since, only positive input (thus, only inhibitor intake) is possible, the applied sinusoidal signal had the following properties: amplitude: $5 \mathrm{mg} / \mathrm{kg}$; offset: $+10 \mathrm{mg} / \mathrm{kg}$; frequency: $1 \mathrm{rad} /$ day.
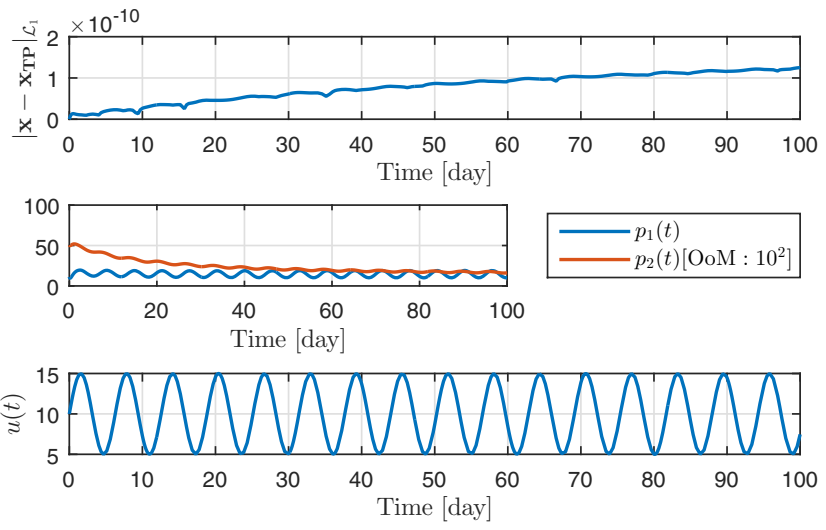

Figure 5. Upper Diagram: Comparison of the states of the transformed model and TP Model I. based on $\mathcal{L}_{1}$ norm, beside sinusoidal input (States: $\mathbf{x}(t)=$ $\left.\left[y_{1}(t), y_{2}(t), g(t)\right]^{\top}\right)$. Lower Diagram: Vary of scheduling parameters over time. OoM: Order of Magnitude.

As it can be seen on Fig. 5 and 6, the differences were only numerical $\left(\approx 10^{-9}\right)$ in both cases between the states of the transformed model and the TP models beside sinusoidal input. Over the 100 day, the steady states of the models were
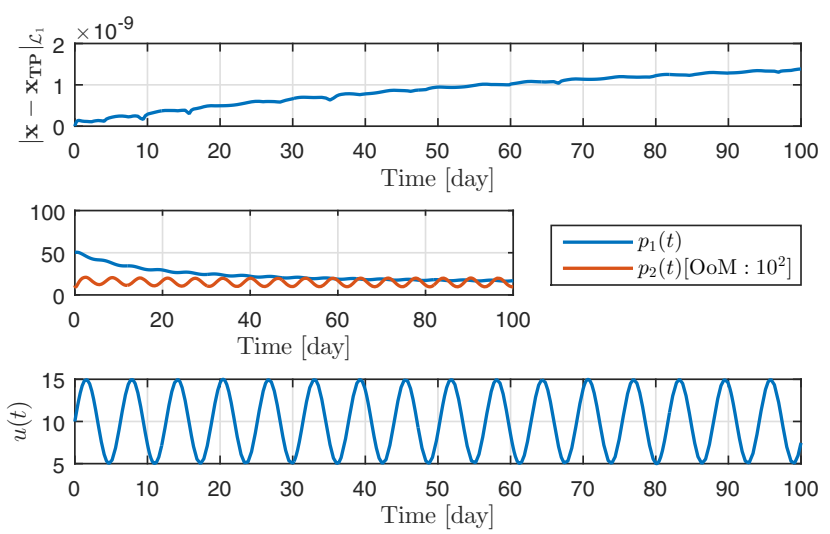

Figure 6. Upper Diagram: Comparison of the states of the transformed model and TP Model II. based on $\mathcal{L}_{1}$ norm, beside sinusoidal input (States: $\mathbf{x}(t)=$ $\left.\left[y_{1}(t), y_{2}(t), g(t)\right]^{\top}\right)$. Lower Diagram: Vary of scheduling parameters over time. OoM: Order of Magnitude.

not reached, however, it became around the day 140 - the order of the differences did not change.

\section{Answer of the Models beside Impulse kind Control Input}

From practical point of view, this test was the most important one, since, similar drug dosage delivery is applied in clinical environment and we would like to use similar strategy regard to the future controller design. As in the previous case, only positive input can be applied. The applied control input had impulse kind nature with the following properties: amplitude: $200 \mathrm{mg} / \mathrm{kg}$; period: $10 \mathrm{mg} / \mathrm{kg}$; pulse width: $1 \%$ of the period.
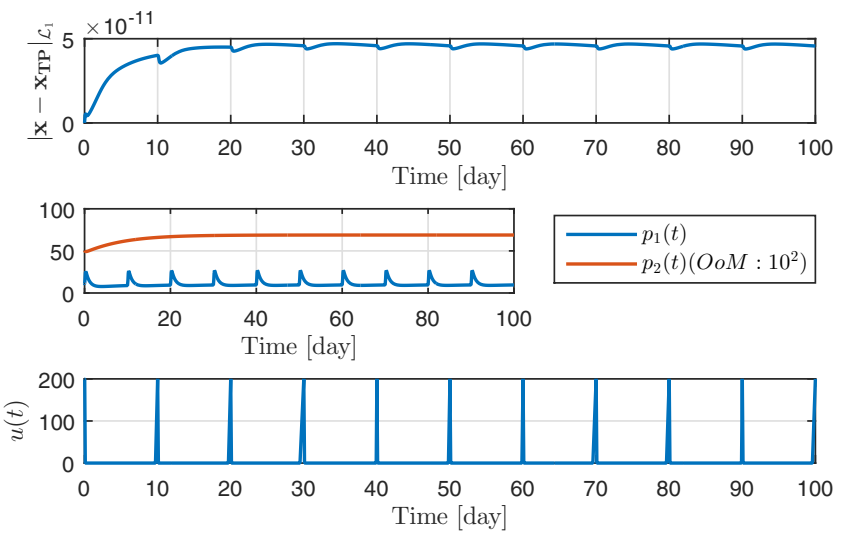

Figure 7. Upper Diagram: Comparison of the states of the transformed model and TP Model I. based on $\mathcal{L}_{1}$ norm, beside impulse kind input (States: $\mathbf{x}(t)=$ $\left.\left[y_{1}(t), y_{2}(t), g(t)\right]^{\top}\right)$. Lower Diagram: Vary of scheduling parameters over time. OoM: Order of Magnitude.

Based on the results - Fig. 7 and 8 -, however the dynamics of both differences were similar and the order of them are only numerical $\left(\approx 10^{-10}\right)$ as well, the highest dissimilarity occurred in this case concerning to the vary of the parameter vectors $\mathbf{p}(t)$ over time. 

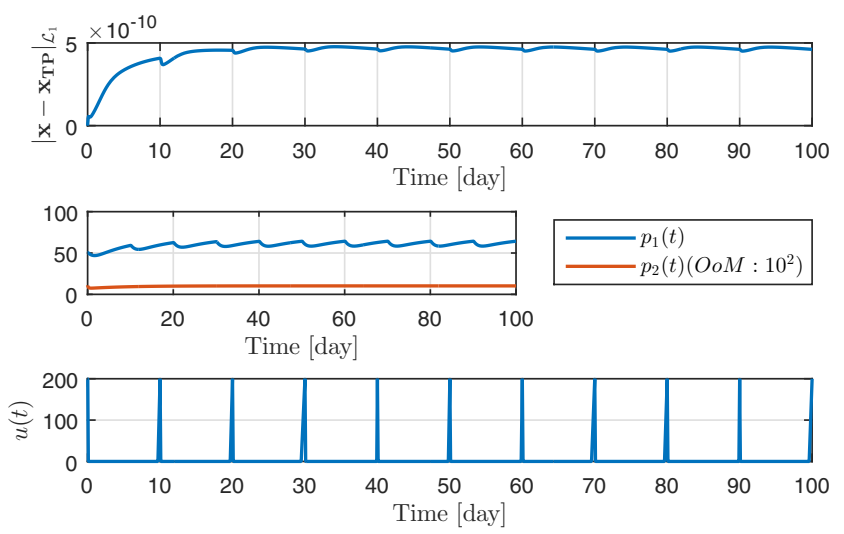

Figure 8. Upper Diagram: Comparison of the states of the transformed model and TP Model II. based on $\mathcal{L}_{1}$ norm, beside impulse kind input (States: $\mathbf{x}(t)=\left[y_{1}(t), y_{2}(t), g(t)\right]^{\top}$ ). Lower Diagram: Vary of scheduling parameters over time. OoM: Order of Magnitude.

\section{CONCLUSION}

In this paper we investigated the applicability of the TP model transformation in case of a transformed tumor model in order to develop such TP models which can be used for control purposes in our future work. We compared the behavior of the transformed model to the developed TP models without external input signal and beside the presence of inhibitor intake. In all cases, we experienced that the TP models approximate the transformed model with high precision and in all cases only numerical deviation occurred between the models. During the numerical simulations, we did not experienced stability problems thank to the applied limitation - although, the introduced operating domain of the states and parameter vector were tighter than the original tumor growth model. Since, the control goal in such kind of tasks is to decrease the volume of the tumor to a maintainable or operable size, the selected limits do not decrease the generality of the TP models - which can be applied for TP-based tumor growth control. In our future work we will compare our results with measurements from animal experiments.

\section{ACKNOWLEDGMENT}

The Authors thankfully acknowledge the support of the Robotics Special College of Óbuda University and the Óbuda University's Research and Innovation Center.

\section{REFERENCES}

[1] N. Vasudev and A. Reynolds, "Anti-angiogenic therapy for cancer: current progress, unresolved questions and future directions," Angiogenesis, vol. 17, no. 3, pp. 471-494, 2014.

[2] B. Al-Husein, M. Abdalla, M. Trepte, D. DeRemer, and P. Somanat, "Anti-angiogenic therapy for cancer: An update," Pharmacotherapy, vol. 32, no. 12, pp. 1095-1111, 2012.

[3] Y. Kubota, "Tumor angiogenesis and antiangiogenic therapy," Keio $J$ Med, vol. 61, pp. 47 - 56, 2012.

[4] D. Drexler, J. Sápi, and L. Kovács, "Potential Benefits of Discrete-Time Controller-based Treatments over Protocol-based Cancer Therapies," ACTA Pol Hung, vol. 14, no. 1, pp. 11-23, 2017.

[5] O. Scharovsky, L. Mainetti, and V. Rozados, "Metronomic chemotherapy: changing the paradigm that more is better," Curr Oncol, vol. 16, no. 2, pp. 7-15, 2009.
[6] T. Browder, C. Butterfield, B. Kraling, B. Shi, B. Marshall, M. O'Reilly, and J. Folkman, "Antiangiogenic scheduling of chemotherapy improves efficacy against experimental drug-resistant cancer," Cancer Res, vol. 60, no. 7, pp. 1878-1886, 2000.

[7] J. Sápi, L. Kovács, D. Drexler, P. Kocsis, D. Gaári, and Z. Sápi, "Tumor volume estimation and quasi-continuous administration for most effective bevacizumab therapy," PLOS ONE, vol. 10, no. 11, 2015.

[8] J. Sápi, D. Drexler, I. Harmati, Z. Sápi, and L. Kovács, "Qualitative analysis of tumor growth model under antiangiogenic therapy: choosing the operating point and design parameters for controller design," Optim Contr Appl Met, vol. 37, p. 848-866, 2015.

[9] J. Sápi, D. Drexler, and L. Kovács, "Comparison of Protocol-based Cancer Therapies and Discrete Controller-based Treatments in the Case of Endostatin Administration," in SMC 2016 - IEEE International Conference on Systems, Man, and Cybernetics. IEEE, pp. 3830-3835.

[10] L. Kovács, "Linear parameter varying (LPV) based robust control of type-I diabetes driven for real patient data," Knowl-Based Syst, vol. 122, pp. 199-213, 2017.

[11] G. Eigner, "Novel LPV-based Control Approach for Nonlinear Physiological Systems ," ACTA Pol Hung, vol. 14, no. 1, pp. 45-61, 2017.

[12] G. Eigner, P. Horváth, J. Tar, I. Rudas, and L. Kovács, "Application of Robust Fixed Point control in case of T1DM," in SMC 2015 - IEEE International Conference on Systems, Man, and Cybernetics. IEEE, p. $2459-2464$

[13] J.K. Tar, J.F. Bitó, L. Nádai, and J.A.T. Machado, "Robust fixed point transformations in adaptive control using local basin of attraction," ACTA Pol Hung, vol. 6, no. 1, pp. 21-37, 2009.

[14] A. Szeles, D. Drexler, J. Sápi, I. Harmati, and L. Kovács, "Modelbased Angiogenic Inhibition of Tumor Growth using Adaptive Fuzzy Techniques," Period Polytech Elec Comput Sci, vol. 58, no. 1, pp. 2936, 2014.

[15] J. Kuti, P. Galambos, and P. Baranyi, "Minimal volume simplex (mvs) convex hull generation and manipulation methodology for tp model transformation," Asian J Control, vol. 19, no. 1, pages = 289 - -301, 2017.

[16] P. Baranyi, Y. Yam, and P. Varlaki, Tensor Product Model Transformation in Polytopic Model-Based Control, 1st ed., ser. Series: Automation and Control Engineering. Boca Raton, USA: CRC Press, 2013.

[17] P. Hahnfeldt, D. Panigrahy, J. Folkman, and L. Hlatky, "Tumor development under angiogenic signaling: A dynamical theory of tumor growth, treatment response, and postvascular dormancy," Cancer Res, vol. 59, pp. $4770-4775,1999$.

[18] J. Klamka, H. Maurer, and A. Swierniak, "Local controllability and optimal control for a model of combined anticancer therapy with control delays," Math Biosci Eng, vol. 14, no. 1, pp. 195-216, 2017.

[19] D. Drexler, J. Sápi, and L. Kovács, "Optimal discrete time control of antiangiogenic tumor therapy [Accepted for presentation]," in IFAC 2017 - 20th World Congress of The International Federation of Automatic Control. IFAC

[20] K. Tanaka and H. O. Wang, Fuzzy Control Systems Design and Analysis: A Linear Matrix Inequality Approach, 1st ed. Chichester, UK: John Wiley and Sons, 2001.

[21] J. Kuti, P. Galambos, and P. Baranyi, "Minimal volume simplex (mvs) approach for convex hull generation in tp model transformation," in 2014 18th International Conference on Intelligent Engineering Systems (INES 2014). IEEE Hungary Section, pp. $187-192$.

[22] K. Wu and Y. Yam, "Control stability of tp model transformation design via probabilistic error bound of plant model," in Proceedings of 2013 IEEE International Conference on Systems, Man, and Cybernetics SMC 2013. IEEE SMCS, 2013, pp. 1259 - 1264.

[23] P. Galambos and P. Baranyi, "TP ${ }_{\tau}$ model transformation: A systematic modelling framework to handle internal time delays in control systems," Asian J Control, vol. 17, no. 2, pp. 1 - 11, 2015. 